\title{
Relating magnetic field strengths to hard $X$-ray emission in solar flares
}

\author{
C. P. Goff ${ }^{1}$, S. A. Matthews ${ }^{1}$, L. van Driel-Gesztelyi ${ }^{1,2,3}$, and L. K. Harra ${ }^{1}$ \\ 1 Mullard Space Science Laboratory, Holmbury St. Mary, Dorking, Surrey RH5 6NT, UK \\ e-mail: cpg1@mssl.ucl.ac.uk \\ 2 Observatoire de Paris, LESIA, FRE 2461 (CNRS), 92195 Meudon Cedex, France \\ 3 Konkoly Observatory, PO Box 67, 1525, Budapest, Hungary
}

Received 5 March 2004 / Accepted 3 May 2004

\begin{abstract}
The observation of hard X-ray (HXR) emission in solar flares provides important diagnostic information about the acceleration and subsequent transport of energetic electrons in the flare process. However, while hard X-rays are thought to be emitted from the flare footpoints through thick-target bremsstrahlung interactions, the details of the transport of accelerated electrons through the solar atmosphere still remains unclear.

Trapping of the electrons is one particular effect that is expected to occur as a result of the convergence of the magnetic field between the corona and the chromosphere. In this case the brightness of the HXR footpoints should be related to the strength of the magnetic field present and we would expect greater precipitation and higher HXR intensities at the footpoints with lower magnetic field strength. This relationship has been observed to hold in many flares (see Sakao 1994) but interestingly the opposite relationship, where the stronger HXR source is found at the stronger magnetic field region, has also been observed in an event studied by Asai et al. (2002).

Using Data from Yohkoh's Hard X-Ray Telescope (HXT) and SOHO's Michelson Doppler Imager (MDI) we have studied the magnetic field strengths at the footpoints of a sample of 32 flares and have compared them to the hard X-ray brightness to determine whether the expected ratios are seen. We find that contrary to the expected relationship the brighter HXR footpoint is found in the region of stronger magnetic field in approximately one third of our sample of events. We discuss the implications of these results in terms of the transport mechanisms.
\end{abstract}

Key words. Sun: flares - Sun: magnetic fields - Sun: X-rays, $\gamma$-rays

\section{Introduction}

Hard X-ray (HXR) emission during a solar flare is a fundamental observation required to improve our understanding of particle acceleration. Commonly the hard X-ray emission is representative of flaring footpoints, the site of non-thermal energy deposition in the lower atmosphere. The flaring loops can be thought of as a representation of a double-ended magnetic bottle which confines non-thermal particles through the Lorentz force, a consequence of the converging magnetic field at the loop ends. These footpoints are rooted in the chromosphere, where the magnetic field is known to be higher than the coronal component at the loop apex, and thus particle trapping is an inevitability.

Trapping is a function of the magnetic mirror ratio $(R=$ $\left.B_{\text {foot }} / B_{\text {apex }}>1\right)$ and also the conservation of angular momentum. The higher $R$, the greater the trapping efficiency, which determines the amount of radiation emitted from particles within the trap. Gyrosynchrotron emission is produced by trapped relativistic electrons, while any leakage determines the level of HXR emission through thick target bremsstrahlung. In solar flares we expect that precipitation will be greatest at the weaker magnetic footpoint, where convergence is smallest while, conversely HXR intensity would be lower in regions of strong magnetic field. This relationship has been observed to hold in flares studied by Sakao (1994). He studied a sample of events where there were two HXR sources which were situated on each side of a magnetic neutral line. He found that four out of five events, which had supporting magnetogram data, had this arrangement, although there was one event, where the opposite was true. This odd event occurred on 2nd November 1991. The magnetogram data for this event however, was approximately eleven hours old by the time of the flare peak. Therefore, it was much more difficult to assess accurately.

This "unusual" behaviour has since been seen by Asai et al. (2002) in a two ribbon flare which was observed on 10th November 1998. In this case the two HXR sources occurred in the strong magnetic regions, whereas the weaker magnetic regions situated under $\mathrm{H} \alpha$ kernels had no HXR emission at all. This behaviour is explained due to a combination of the dynamic range of Yohkoh's Hard X-ray Telescope (HXT) 
and the estimated energy release rates at the regions with HXR sources, which are much larger than in the kernels without HXR emission.

Aschwanden et al. (1999) looked at 54 Yohkoh flares, where there were 2 footpoints. They found there was no significant energy dependence of the HXR asymmetry, but found that the asymmetry is inversely correlated to the trapping efficiency. By combining their observations and calculations they predicted that the median value of the ratio between the field strengths at each footpoint would be 1.2. They did not, however, have magnetogram data to test their theory.

It was on the basis of both the Asai et al. (2002) result and the work of Aschwanden et al. (1999) that we examine further examples. It was important to establish a reliable and effective method of measuring footpoint field strengths and to be able to effectively compare these to HXR intensities. With a method established one can explore the catalogue of events and determine the frequency of these odd types of events with much more accuracy, and additionally the potential causes of these events.

\section{Observations and analysis}

A sample of 32 flares ranging from C-class to X-class has been taken between 1996 (the launch of the Solar and Heliospheric Observatory, $\mathrm{SOHO}$ ) and the end of 2001. The events were chosen such that they consisted of just two HXR sources both sides of the magnetic neutral line and had to be within $40^{\circ}$ of the disc centre. Within this $40^{\circ}$ region, assuming a radial field, the line of sight effects are minimised allowing for a more accurate measurement of magnetic field. We use data from Yohkoh's Soft X-Ray Telescope (SXT; Tsuneta et al. 1991), which images soft $X$-rays through a grazing incidence telescope and four filters covering the energy range $0.25-4 \mathrm{keV}$ and Yohkoh's Hard X-Ray Telescope (Kosugi et al. 1991) for observations in the energy range of $15-100 \mathrm{keV}$. HXT is a Fourier synthesis type imager, capable of measuring X-rays from nonthermal electrons and super-hot plasmas in four discrete energy channels. These bands are: $L(14-23 \mathrm{keV}), M 1(23-33 \mathrm{keV})$, $M 2(33-55 \mathrm{keV})$ and $H(53-93 \mathrm{keV})$ with a best angular resolution of $\approx 5$ arcsec and temporal resolution of $0.5 \mathrm{~s}$. Additionally we study magnetograms from the Michelson Doppler Imager (MDI; Scherrer et al. 1995) on SOHO.

When choosing the events it was important that there were data in the $L, M 1$ and $M 2$ channels of HXT which showed the impulsive phase well above the background level. This would allow spectral information to be determined along with the reconstruction of accurate images up to the $M 2$ channel which are used to give a footpoint location and intensity. Images were reconstructed from a set of the 64 spatially modulated photon counts accumulated to a minimum of 200 in $M 2$ and then using a code based on that of Sakao (1994) which uses the Maximum Entropy Method (MEM) (Gull \& Daniell 1978) to reconstruct the image. MEM works by maximising a quantity called entropy within the constraints of the observations. The main advantage of this method is that it chooses the smoothest solution consistent with the data, produces positive images and handles Poisson errors well. The process uses the location of the SXT images as a reference to ensure that the position of the reconstruction is as precise as possible. The SXT images are prepared by flagging saturated pixels, while removing spacecraft jitter and correcting for roll. With this sample we may determine any relationship between magnetic field strength and HXR intensity.

\subsection{Analysis method}

With a suitable sample of events, it is then possible to study the mean magnetic field strength beneath each flare footpoint. It was expected that a weaker HXR source would be discovered in the region of stronger magnetic field as the magnetic mirror inherent in a converging magnetic field would impede the cascade of electrons responsible for this emission through the process of thick-target bremsstrahlung.

When looking at a sample like this with the intention of making a direct comparison between different events it is fundamental that the magnetic field strength beneath the flare footpoints is determined in a systematic and reliable manner. There are clearly many uncertainties involved (Sect. 2.2) and steps must be taken to minimise their effects. Any offsets between images could give false results which would make comparison unreliable. Therefore to prepare a reliable method for measuring the magnetic field strength each individual HXR source was treated as the footpoint. MDI images, which have been differentially rotated to the time of HXR emission and have been corrected for Earth-view, were overlaid with HXR intensity contours from the $M 2$ channel in increments of $10 \%$ of the peak intensity. The mean single polarity magnetic field strength within each contour could then be obtained and the sum of the mean magnetic field values at each contour level across the whole sample were then plotted to give Fig. 1. This plot shows clearly that with increasing HXR intensity the mean magnetic field strength increases as fewer and fewer low valued pixels are included. At the $60 \%$ contour levels the mean magnetic field strength begins to drop. As the number of pixels decreases so too does the area over which the field is determined. Thus any offset between the two images becomes highly influential. Hence we chose the $60 \%$ contour level as being representative of the mean magnetic field strength under the source.

For this study the HXR intensities were determined from the $20 \%$ contour (Sato et al. 1999). Although this is a physically larger region than that used for the magnetic field strength it is important to include all reliable counts. This represents most of the relevant counts, which one can say come from the specific source with confidence. The $20 \%$ level is determined from the reciprocal of the dynamic range which has been empirically estimated to be 10-20\% (Kosugi et al. 1992). A smaller region can be used for the magnetic measurements as here it is the positional information which is important.

\subsection{Sources of uncertainty}

One of the significant sources of uncertainty in these results comes from the alignment between the two types of images. The pointing stability of Yohkoh is thought to be very good and 


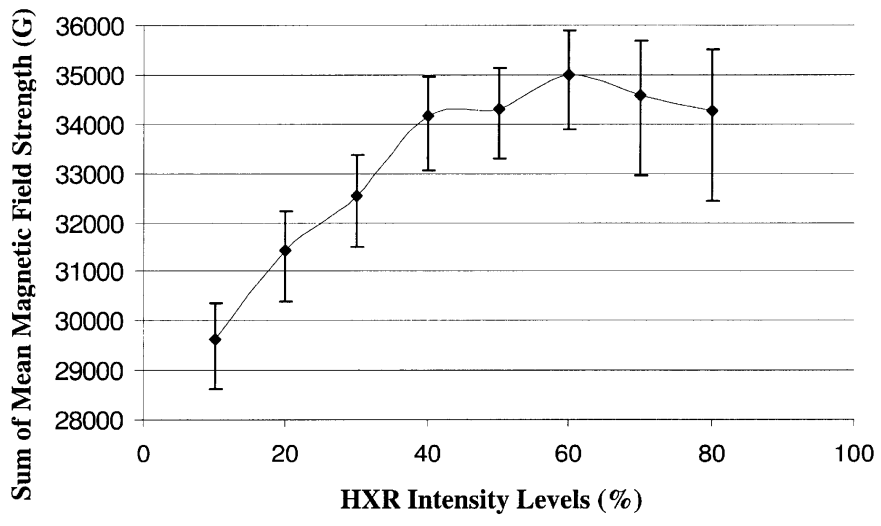

Fig. 1. Each HXR contour level defines a region of magnetic field over which the mean magnetic field strength is determined. This plot is a representation of the unsigned magnetic field variation with a percentage of the HXR intensity in a flare footpoint, as defined by Yohkoh's HXT $M 2$ channel MEM images. It is determined by the sum of the mean magnetic field strength at each level across the whole sample.

of the order of 1" (Ogawara et al. 1991). Therefore we assume the discrepancy between this and the MDI magnetograms is up to one MDI pixel, which is about $2^{\prime \prime}$ around the disc centre. So in each case the HXR images were shifted $2^{\prime \prime}$ in the direction of maximum difference. The mean magnetic field strength under each footpoint was then measured again. The difference between the previously measured value and this maximum shifted value was taken as the uncertainty. This however, does not fully address projection effects due to the line of sight measuring techniques, but is the best that can be achieved with the information that exists. The values recorded by MDI around disc centre should be accurate, but line of sight limitations mean that they are really a lower limit.

The work of Hagenaar et al. (2002) has demonstrated the importance of MDI noise. This noise level is expected to be 3.2 $\mathrm{G}$ and has been subtracted from all of the values used in this study. Additionally it is now thought that MDI can dramatically underestimate the field strength measurements. Berger \& Lites (2002) reported that actual values could be lower on average by a factor of $0.64 \pm 0.013 \mathrm{G}$. However, this is based on a single set of observations and has not yet been verified for all observations. Thus any values quoted throughout this paper are uncorrected for this, but the reader should keep this in mind.

\section{Results}

Using the above method of alignment the individual footpoints of each of the flares had their HXR intensity recorded along with their associated mean magnetic field strength. These two values are plotted against each other in Fig. 2.

It was expected that there would be some inverse relation, which is implied by the work of Sakao (1994). His data demonstrated that the stronger HXR emission was from a region of weaker magnetic field strength in four out of the five featured cases from the $\mathrm{M}$ to $\mathrm{X}$-Class range. With the large scatter seen in Fig. 2 any such pattern is not clear. This plot does, however, demonstrate the complexity of this study.

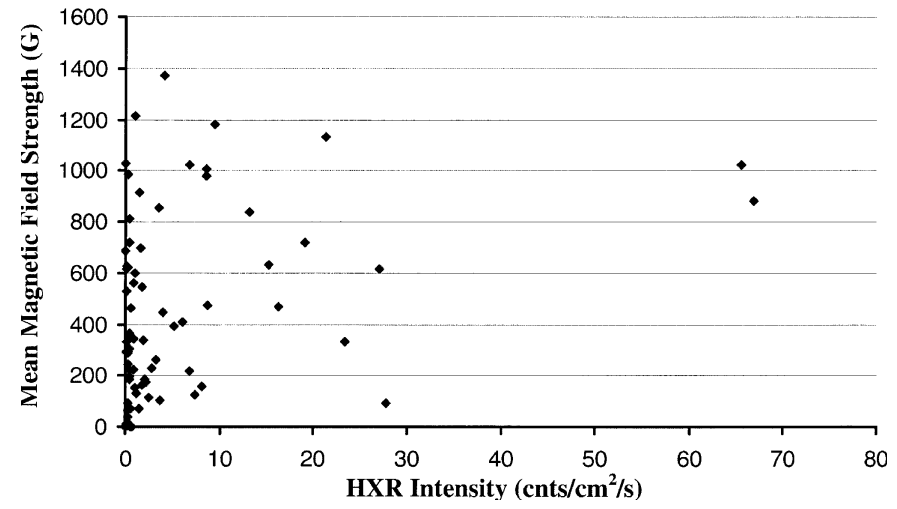

Fig. 2. The HXR intensity at the $60 \%$ intensity level was determined and can be seen here plotted against the unsigned mean magnetic field strength beneath it. It is clear that there is wide variation in all values possibly an effect of comparing many different flare magnitudes.

Our sample covers a range of flares in $\mathrm{C}$ through to X-Class. In any given flare with two HXR sources, the magnitude of the magnetic field beneath each can be very different. If one changes the plot in Fig. 2 to include flares from only a single GOES classification there is little difference in the appearance other than to simply reduce the number of points plotted as the large scatter remains.

To limit the effects of this wide variation due to the flare magnitudes, ratio values were used. These ratios are determined using observations under each footpoint in a particular flare which gives a value which can be used in direct comparison regardless of flare energy, assuming both footpoints have a mean magnetic field strength well above zero. If the source is small in area and has a very low field strength any alignment offset plays a proportionately higher role and as such the confidence in the value falls, leading to a ratio which is artificially high. The added benefit with this ratio method is that any underestimation of field has less of an effect, assuming these effects are linear. Thus the HXR and magnetic field strength ratios can be plotted against each other, the result of which is seen in Fig. 3. This plot is such that the stronger HXR source is always divided by the weakest, resulting in HXR ratios which are always greater than unity. It is apparent that some $47 \%$ (15) of events have both of their ratios greater than one, a regime in which the stronger HXR source is situated in a region of stronger magnetic field. This is unexpected as in Sakao's (1994) sample of events, it was found to be the case that the stronger HXR source was located in a region of lower magnetic field strength, which is represented by the lower region on the plot in Fig. 3; a consequence that would be expected as a result of simple magnetic convergence and mirroring.

Instead, we find there are two distinct groups: those whose magnetic field ratio is less than one where the flare follows the Sakao relation (Sakao, S-type events) and those greater than one where they do not (non-Sakao, N-type events). Due to the uncertainties inherent in the measurement technique it is reasonable to assume that those events where the error bars cross $y=1$ (the symmetry line) are likely to be the almost symmetrical in magnetic flux density, and as such should be thought of as an additional sub-group (balanced, B-type events). 


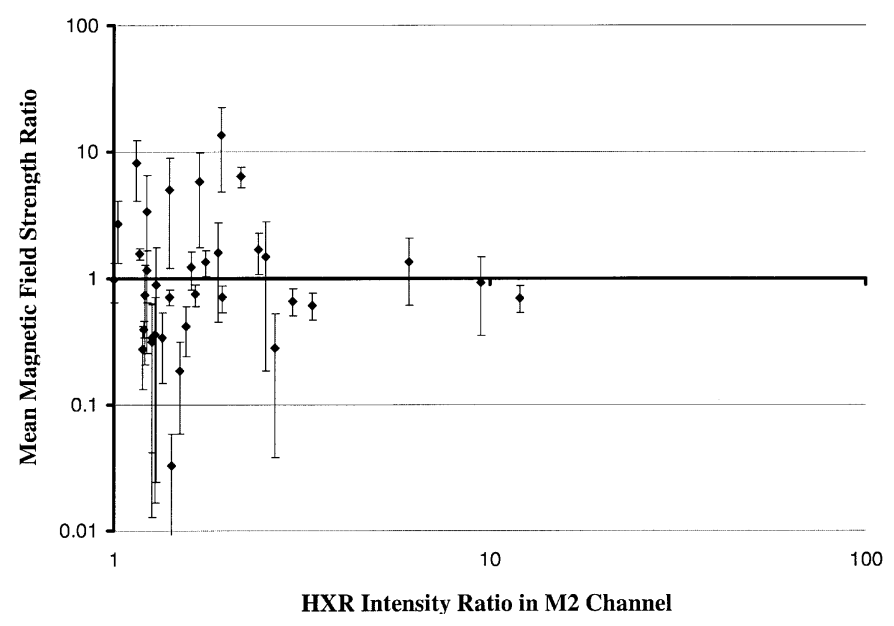

Fig. 3. The HXR intensity ratio between the brightest and weakest footpoints in any given flare from the $M 2$ channel are plotted directly against the mean unsigned magnetic field strength ratio between the same footpoints. The region below the $y=1$ line represents by the S-type events (those events where the stronger HXR footpoint lies in the region of weaker magnetic field) and the region above the $\mathrm{N}$-type (where the stronger HXR lies in a region of stronger magnetic field).

By forming this additional group, we are simply identifying the events where measurement uncertainties may play a dominant role and removing them from the sample.

The result in Fig. 3 was determined by looking at the double HXR sources in the $M 2$ channel at the peak of activity. On closer inspection we find additional complications. One can compare the SXR light curves for regions situated over coincident regions to the HXR footpoints. If these footpoints are connected by a single SXR loop it is reasonable to assume that the plasma at each end would have similar properties. Thus the shape of the light curves should be similar. If the shape differs substantially from one region to another it may indicate that the regions are not connected in such a simple way and as such the possibility of different structures or magnetic connectivities should be considered. This possibility would indicate that there may be significant information beyond the resolution of our observations and as a result they should be removed from consideration.

In our sample five events have SXR light curves in these regions which indicate there is a possibility that the flare may be the result of an interaction with a small loop, possibly emerging from beneath (as in Hanaoka's (1996) interacting loop studies) a larger loop. In these cases at least one of the HXR sources may actually be the unresolved footpoints of this small loop and as a result may not fit within the confines of this study. In summary of these events, two have their error bars crossing the symmetry line (B-type) and three are from the N-type events reducing this group to just seven. When we exclude the above problematic events we have eight B-type events, 14 S-type and seven N-type events. This therefore reduces the ratio between the two non-symmetric groups as $2: 1$.

Further to this, in 11 cases we had sufficient data to look at the timings in the HXR emission between the two sources. If we first look at the N-type events, we find there are two events where we can look at the light curves for each individual footpoint. In both cases the emission appears simultaneous. However, these are compact flares and it may not be possible to measure the time difference given the limited distance.

In the S-type category seven events have sufficient data to look at the individual light curves. In four of these the two individual light curves peaked simultaneously, within the limits of the data. In the other cases the footpoint with the strongest HXR source had a light curve that peaks first. This may suggest that the acceleration site of the electrons is closer to the site of the stronger footpoint as there is a longer flight time to reach the weaker footpoint. Alternatively it may suggest that the electrons precipitating towards the stronger footpoint are less impeded in their journey.

In an attempt to understand the role of the magnetic environment the distribution of magnetic flux density under each of the flare footpoints was observed in a qualitative manner. We attempted to see if the distribution could have an impact in the type of event observed. It was thought that if there was high convergence in the magnetic field there would be a rapid change or peak in the flux density as you cross the footpoint, whereas if there was less convergence then there would be more of a flat distribution. There was however, nothing to distinguish between the two groups of events. Often the footpoint may only be 3 or 4 MDI pixels across and this would not allow any distinction to be determined between these types of distributions. However, there is an added complication with this approach and that is your viewing angle. Without a clear knowledge of one's viewing angle and the directions of the magnetic field you cannot be sure that your observations are real and not simply a product of your point of view.

\subsection{Spectral evolution}

In addition to the above properties the spectral evolution was measured. Differences in this evolution can give insight into the trapping of electrons in a flare. It was hoped that these measurements may give a clue as to why the N-type events exist or how they differ from the S-type. Typically spectral evolution in solar flares follows the soft-hard-soft pattern first recognised by Parks \& Winckler (1969); this pattern was evident across all of the above flare types.

In order to study this it was important that there were data in at least the first three channels of HXT. Background was subtracted and a single power-law photon spectrum was assumed. The spectral flux is assumed to be proportional to the photon energy $\left(E^{-\alpha}\right)$ where $\alpha$ is the spectral index. In each flare the spectral evolution was observed and recorded at its hardest. The mean value for each group was ascertained along with the standard deviation in the spread. The S-type events were found to have a mean value of 3.4 with a deviation from the mean of 1.4, while the N-type had a value of 3.5 with a deviation of 1.2. Even the B-type events had a mean of 3.3 with a deviation of 0.7 . Unfortunately there were not enough events where we could study the evolution in each of the flare footpoints, which would have given us much more information. We therefore found no quantitative difference which may indicate different transport or trapping of electrons in this limited dataset. 


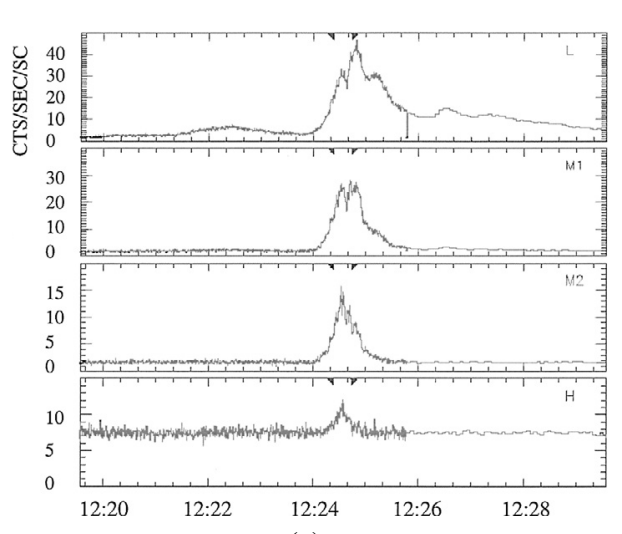

(a)

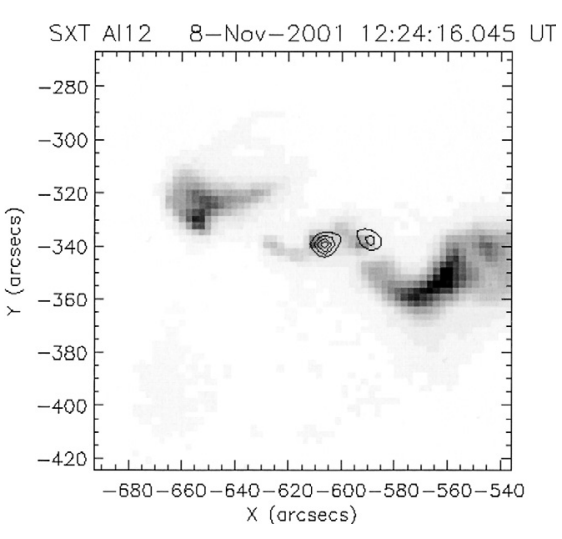

(b)

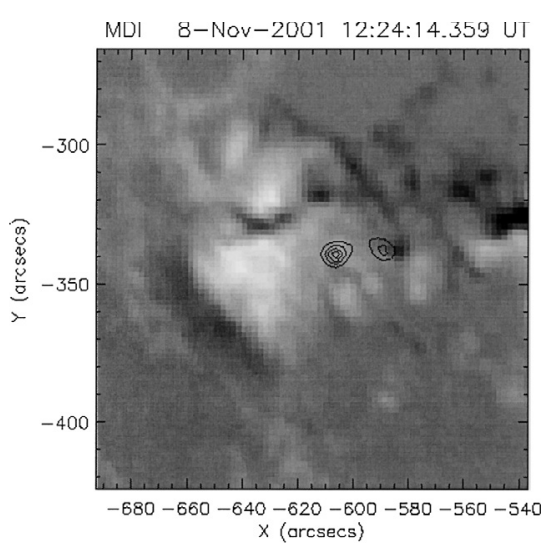

(c)

Fig. 4. a) HXT light curves for the flare on 8th November 2001 across each of the four energy channels; b) the greyscale image represents a full resolution SXT image of the 8th November 2001 event at 12:24 UT in the Al12 filter. Superimposed are the HXR intensity contours 12:24:30 UT at 20\%, 40\%, 60\% and $80 \%$ of peak intensity in $M 2$ channel; c) the greyscale image represents the magnetogram (where black is negative) from the 8th November 2001 event at 12:24 UT with the same HXR intensity levels as in b) superimposed. It is clear that the footpoints are situated in regions of opposite polarity as expected.

\subsection{Examples}

We now consider some specific examples to illustrate the different types and their properties. We shall look at an S-type, an S-type where the HXR ratio is very high, a B-type and an N-type event.

\subsubsection{S-type event, 8th November 2001}

This event occurred around 12:24 UT on the 8th November 2001 and the HXR light curves can be seen in Fig. 4a. They show the total emission in each of the four HXT energy channels.

Figure 4b shows a soft X-ray image over which is superimposed HXR contours from the $M 2$ channel around the time of peak emission in $M 2$. The region is very complex in soft $\mathrm{X}$-rays although the situation is clarified when it is compared to the magnetogram in Fig. 4c. It is part of a large and aging active region and as a result multiple connections are probable. However, it is still apparent that the HXR sources form at the footpoints of a smaller loop, which becomes brighter with time. When the spectral index was determined for this event, it evolved in the classical soft-hard-soft manner and had a value of 3.5 at the hardest.

When the footpoint brightness and mean magnetic field strengths are calculated it is clear that the brightest footpoint forms in the region with the weakest magnetic field strength, as is expected from the results of Sakao (1994). The ratio between the footpoint brightness is 3.0 and the ratio between the footpoint magnetic flux densities is 0.29 .

Further details of the remaining Sakao type events can be seen in Table 1.

\subsubsection{High ratio S-type event, 28 July 1999}

This event occurred around 08:30 UT on the 28th July 1999 UT and the HXR light curves can be seen in Fig. 5a. They show the total emission in each of the four HXT energy channels. The light curves in this case are more gradual, and long lived. Also the peak spectral index for this flare was only 5.5, one of the softer events.

The SXT image shown in Fig. $5 b$ is superimposed with the HXT contours from the M2 channel. The two HXR sources clearly coincide with the footpoint regions of a compact flaring loop as expected. The eastern source is brighter in hard X-rays than the western source by a factor of twelve. This is unexpectedly high, but can be explained by the relatively weak nature of the western source. There are only five pixels in this source with intensity greater than $40 \%$ of the maximum intensity.

However, the soft X-ray images allow the analysis of this plasma at each end of a loop. Each region which appears coincident with the HXR emission has a very similar light curve and makes it likely that the two HXR sources are true conjugate footpoints. It was possible in this event to take light curves of each of the HXR footpoints, and the emission occurs simultaneously, within the limits of the data as shown in Fig. 6. However, it is also possible that the image cadence is insufficient to give a real indication of which footpoint peaks first.

If the HXR image is superimposed onto a rotated and coaligned magnetogram from MDI we see that the footpoints are either side of the magnetic neutral line. This is clearly seen in Fig. 5c. There is nothing in this event which clearly distinguishes it from the other events other than the very high ratio between the HXR intensities.

\subsubsection{B-type event, 8th April 2000}

This event occurred at around 02:38 UT on the 8th April 2000. The HXR light curves can be seen in Fig. 7a. They show a rapid increase in emission during the early phase of the flare but a more gradual decline in emission as time progresses. The peak spectral index for this flare is 3.1 which is very close to the mean value of 3.3 in the B-type events. 
Table 1. Featured properties of the S-type events.

\begin{tabular}{lcclcc}
\hline \hline Date & Time (UT) & Goes class & Position $(\operatorname{arcsec})$ & HXR ratio & Magnetic ratio \\
\hline 28-Jul.-1999 & $08: 31$ & M 2.3 & $(-55.1,-331.7)$ & 12.1 & $0.7_{-0.16}^{+0.16}$ \\
22-Dec.-1999 & $10: 54$ & C 6.3 & $(-382.8,404.7)$ & 1.3 & $0.3_{-0.19}^{+0.00}$ \\
06-Jul.-2000 & $20: 41$ & C & $(-258.2,254.2)$ & 1.2 & $0.4_{-0.06}^{+0.05}$ \\
17-Jul.-2000 & $20: 25$ & M 2.4 & $(-553.9,-279.1)$ & 1.2 & $0.3_{-0.14}^{+0.10}$ \\
22-Nov.-2000 & $16: 20$ & C 7.0 & $(-242.6,267.2)$ & 1.3 & $0.3_{-0.11}^{+0.29}$ \\
24-Nov.-2000 & $15: 08$ & X 2.3 & $(166.4,283.9)$ & 1.9 & $0.7_{-0.13}^{+0.17}$ \\
24-Nov.-2000 & $21: 53$ & X 1.8 & $(237.7,291.6)$ & 1.6 & $0.4_{-0.18}^{+0.14}$ \\
06-Apr.-2001 & $01: 47$ & C 7.7 & $(-607.1,-294.5)$ & 1.6 & $0.7_{-0.14}^{+0.10}$ \\
12-Apr.-2001 & $10: 17$ & X 2.0 & $(596.6,-274.2)$ & 1.4 & $0.7_{-0.10}^{+0.10}$ \\
15-Jun.-2001 & $10: 27$ & M 6.3 & $(-557.1,-430.5)$ & 1.3 & $0.3_{-0.00}^{+0.30}$ \\
23-Oct.-2001 & $02: 16$ & M 6.5 & $(-180.8,-389.5)$ & 1.5 & $0.2_{-0.13}^{+0.01}$ \\
31-Oct.-2001 & $08: 05$ & M 3.2 & $(24.1,138.4)$ & 3.4 & $0.6_{-0.14}^{+0.04}$ \\
08-Nov.-2001 & $12: 25$ & M 1.3 & $(-601.7,-359.8)$ & 2.7 & $0.3_{-0.18}^{+0.24}$ \\
11-Nov.-2001 & $10: 57$ & M 1.4 & $(-71.2,-335.9)$ & 3.0 & $0.7_{-0.14}^{+0.15}$ \\
\hline
\end{tabular}

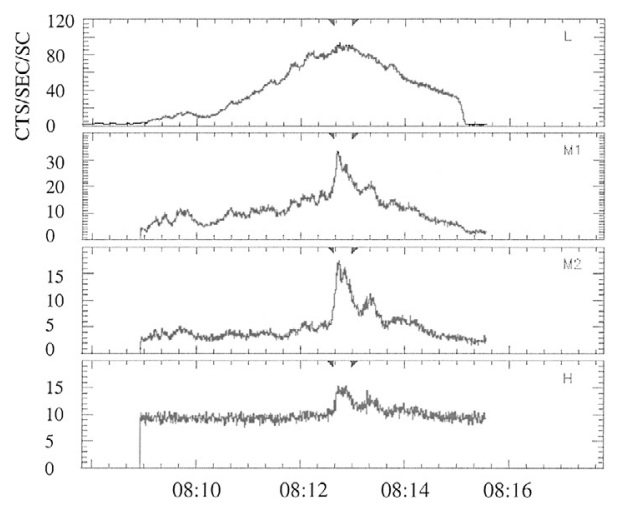

(a)

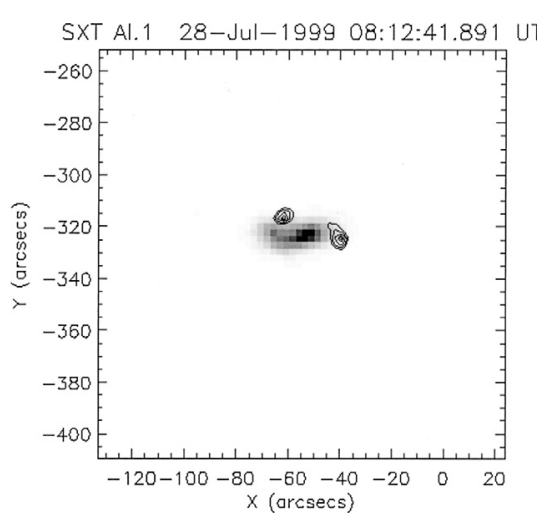

(b)

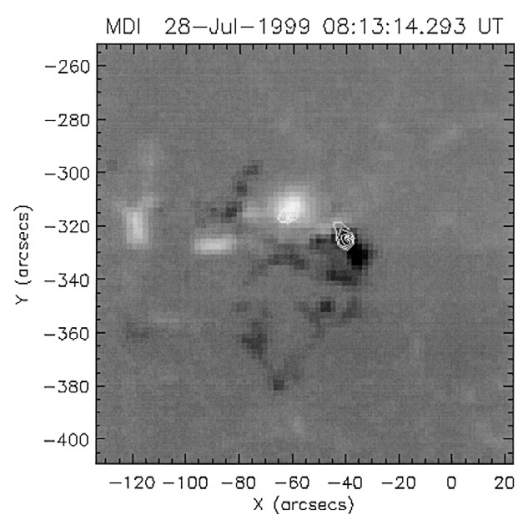

(c)

Fig. 5. a) HXT light curves for the flare on 28th July 1999 across each of the four energy channels; b) the greyscale image represents the full resolution frame SXT image of the 28th July 1999 event at 08:12 UT in the Al.1 filter. Superimposed are the HXR intensity contours 08:12:45 UT at 20\%, 40\%, 60\% and $80 \%$ of peak intensity in $M 2$ channel; c) the greyscale image represents the magnetogram (where black is negative) from the 29th July 1999 event at 08:13 UT with the same HXR intensity levels as in b) superimposed. It is clear that the footpoints are situated in regions of opposite polarity as expected.

In Fig. $7 \mathrm{~b}$ the HXR contours from the $M 2$ channel have been placed over the SXR image. Again, it is clear that the two sources are situated at either end of a flaring loop. These same contours were also placed over the magnetogram in Fig. 7c and each source can be seen to lay in a different magnetic polarity. In this case the magnetic ratio between the two footpoints is $0.9_{-0.00}^{+0.86}$ which would suggest that it is an S-type event, as discussed above. However, the uncertainties described previously, when applied to this event, are highly influential and give rise to the possibility of the ratio actually being greater than unity. This would have the effect of defining the event as N-type rather than S-type as the ratio alone would indicate. There have been no other properties found that distinguish this event, but the limitations imposed on the measurement of the magnetic field strength make a more precise result impossible. It may be that this, along with the other events in this category, is more or less symmetrical in its footpoint field strengths or it may be that the geometry of the event is such that the limitations of line of sight measurements are more apparent. However, as the data stands we cannot accurately place this event along with the others in the group in either the $\mathrm{N}$ - or S-type categories.

Table 2 list the other flares in the B-type group identified through this study.

\subsubsection{N-type event, 16 th March 2000}

This event occurred on 16th March 2000 at around 18:35 UT. From Fig. 8a it can be seen that there are two peaks in the $M 1$ and $M 2$ channels. The $\mathrm{L}$ channel is more gradual as one would expect, but still has two bumps coinciding with the peaks in the higher channel. Unfortunately there is only data up to this second peak and it is impossible to analyse this event beyond that point, although the flare still has only two HXR sources. It should be noted that this double peak is not a characteristic of the N-type events, only a feature of this one. The two sources can be seen in Fig. 8b along with the relation with the soft 

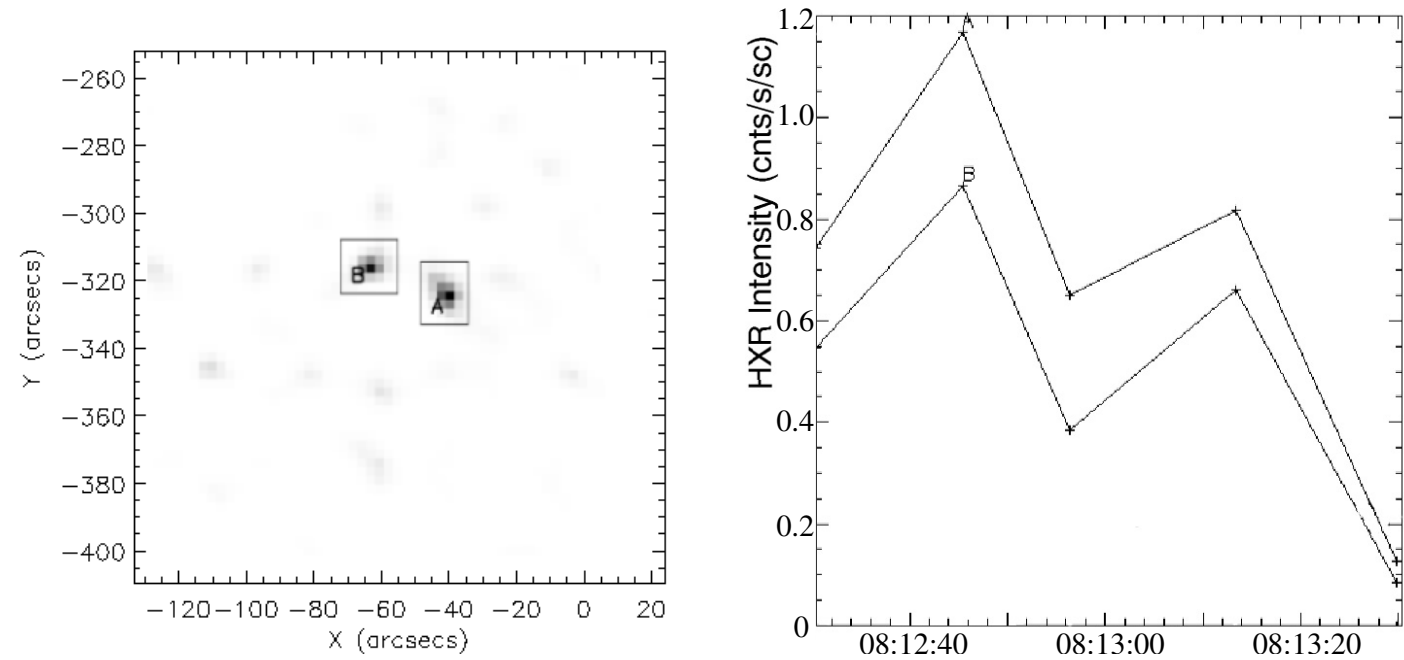

Fig. 6. The M2 HXT image of the 28th July 1999 event at 08:12:45 is shown. Two boxes have been used to select the individual footpoints allowing us to observe the individual light curves seen at the right of this figure. The simultaneous nature of these light curves is clear.

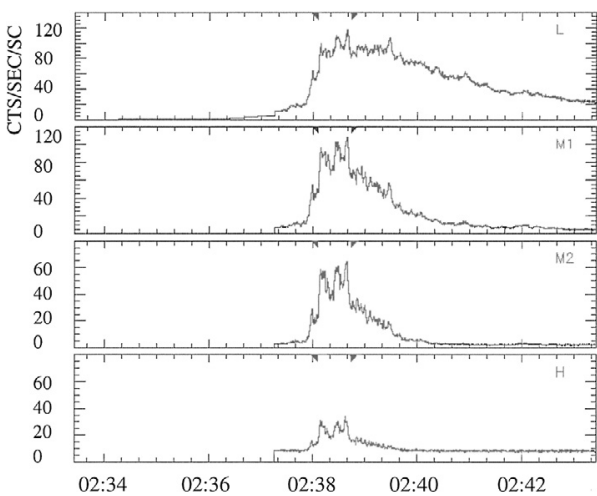

(a)

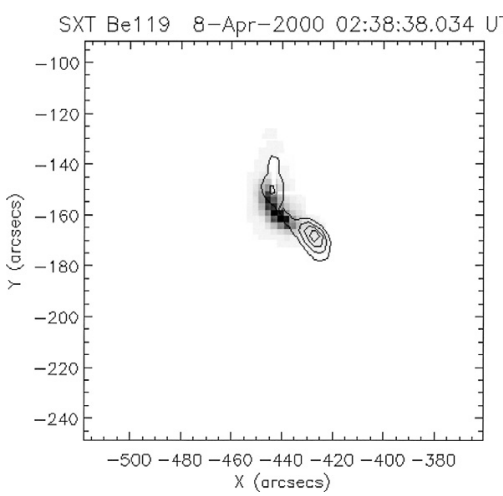

(b)

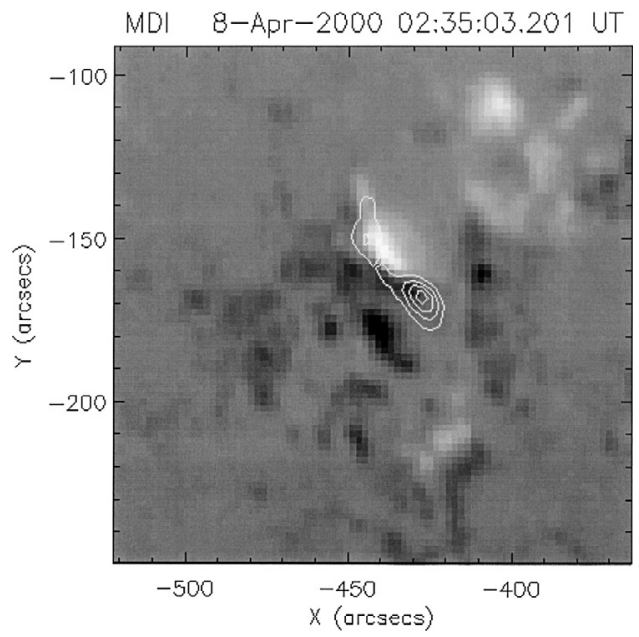

(c)

Fig. 7. a) HXT light curves for the flare on 8th April 2000 across each of the four energy channels; b) the greyscale image represents the full resolution frame SXT image of the 8th April 2000 event at 02:38 UT in the Be119 filter. Superimposed are the HXR intensity contours 02:38:32 UT at $20 \%, 40 \%, 60 \%$ and $80 \%$ of peak intensity in $M 2$ channel; c) the greyscale image represents the magnetogram (where black is negative) from the 8th April 2000 event at 02:35 UT with the same HXR intensity levels as in b) superimposed. It is clear that the footpoints are situated in regions of opposite polarity as expected.

Table 2. Featured properties of the B-type events.

\begin{tabular}{lcclcc}
\hline \hline Date & Time (UT) & Goes class & Position $(\operatorname{arcsec})$ & HXR ratio & Magnetic ratio \\
\hline 30-Jun.-1999 & $11: 26$ & M 1.9 & $(108.8,-294.7)$ & 1.6 & $1.2_{-0.01}^{+0.40}$ \\
25-Aug.-1999 & $01: 35$ & M 3.6 & $(-183.5,-550.7)$ & 1.2 & $0.7_{-0.52}^{+0.35}$ \\
08-Apr.-2000 & $02: 38$ & M 2.0 & $(-440.8,-172.2)$ & 1.3 & $0.9_{-0.00}^{+0.86}$ \\
08-Apr.-2000 & $20: 44$ & M 1.8 & $(-292.3,-155.1)$ & 1.2 & $1.1_{-0.50}^{+0.00}$ \\
25-Nov.-2000 & $18: 38$ & X 1.9 & $(383.9,292.9)$ & 6.1 & $1.3_{-0.20}^{+0.72}$ \\
10-Apr.-2001 & $05: 19$ & X 2.3 & $(125.7,-282.9)$ & 1.0 & $0.9_{-0.33}^{+0.27}$ \\
14-Sep.-2001 & $01: 35$ & M 3.7 & $(-580.2,-320.7)$ & 1.9 & $1.6_{-0.62}^{+1.13}$ \\
17-Sep.-2001 & $08: 21$ & M 1.5 & $(-86.9,-342.6)$ & 2.5 & $1.5_{-0.58}^{+1.29}$ \\
\hline
\end{tabular}

$\dagger$ These events are thought to be complicated by the interaction of multiple SXR loops and thus contributions from unresolved HXR footpoints can not be ruled out.

$\$$ This is the event studied by Asai et al. (2002) and the table lists magnetic footpoint ratios consistent with her observations. 


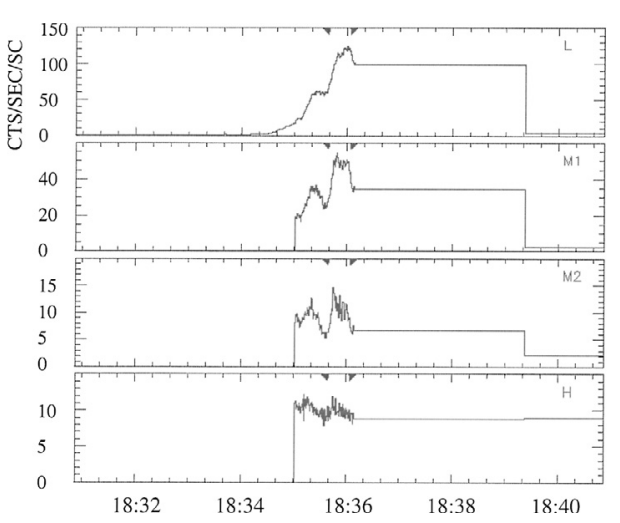

(a)

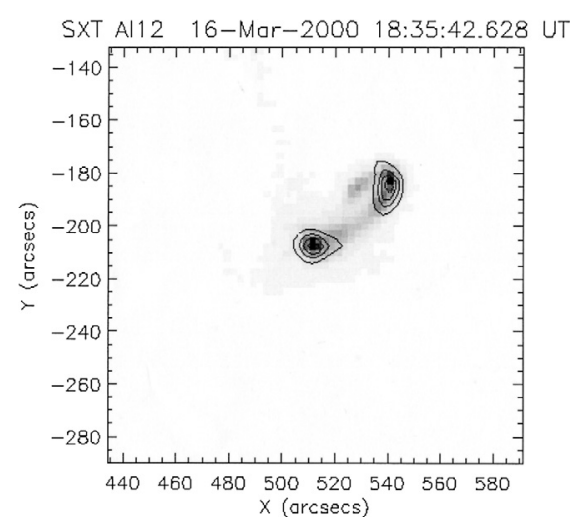

(b)

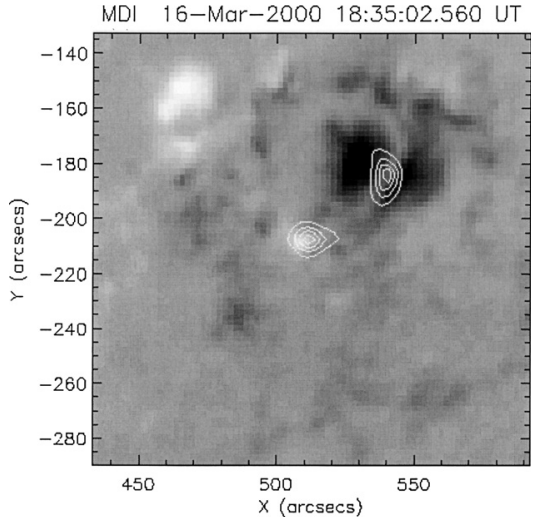

(c)

Fig. 8. a) HXT light curves for the flare on 16th March 2000 across each of the four energy channels; b) the greyscale image represents the full resolution frame SXT image of the 16th March 2000 event at 18:35 UT in the Al12 filter. Superimposed are the HXR intensity contours 18:35:45 UT at 20\%, 40\%, $60 \%$ and $80 \%$ of peak intensity in $M 2$ channel; c) the greyscale image represents the magnetogram (where black is negative) from the 16th March 2000 event at 18:35 UT with the same HXR intensity levels as in b) superimposed. It is clear that the footpoints are situated in regions of opposite polarity as expected.

Table 3. Featured properties of the N-type events.

\begin{tabular}{lcclcc}
\hline \hline Date & Time (UT) & Goes class & Position $(\operatorname{arcsec})$ & HXR ratio & Magnetic ratio \\
\hline 18-Jan.-2000 & $22: 06$ & C 4.3 & $(-255.6,356.1)$ & 1.1 & $8.2_{-4.17}^{+4.75}$ \\
02-Mar.-2000 & $08: 24$ & X 1.1 & $(749.9,-226.6)$ & 1.2 & $1.6_{-0.16}^{+0.00}$ \\
15-Mar.-2000 & $05: 41$ & C 4.0 & $(227.7,-176.7)$ & 1.9 & $13.6_{-8.78}^{+8.77}$ \\
16-Mar.-2000 & $18: 36$ & C 9.0 & $(512.9,-211.7)$ & 1.0 & $2.7_{-1.61}^{+1.36}$ \\
25-Jul.-2000 & $02: 48$ & M 8.0 & $(125.1,-15.1)$ & 1.8 & $1.3_{-0.17}^{+0.30}$ \\
25-Sep.-2000 & $02: 09$ & M 1.8 & $(257.6,55.9)$ & 1.8 & $1.3_{-1.18}^{+0.64}$ \\
06-Apr.-2001 & $19: 16$ & X 5.6 & $(-493.8,-262.9)$ & 2.4 & $1.7_{-0.34}^{+0.60}$ \\
24-Apr.-2001 & $06: 58$ & M 3.1 & $(-223.6,382.9)$ & 1.4 & $5.0_{-3.68}^{+3.85}$ \\
31-Aug.-2001 & $10: 38$ & M 1.6 & $(-553.3,147.2)$ & 1.2 & $3.4_{-3.11}^{+2.88}$ \\
10-Sep.-2001 & $05: 13$ & C 8.7 & $(-214.2,-503.1)$ & 1.7 & $5.8_{-4.03}^{+2.44}$ \\
\hline
\end{tabular}

$\dagger$ These events are thought to be complicated by the interaction of multiple SXR loops and thus contributions from unresolved HXR footpoints can not be ruled out.

X-ray emission. Again these two sources are situated at each end of a flaring loop although the loop is longer than that of the high ratio S-type event, and there appears to be an extra component to this loop near the northern footpoint in a region of single magnetic polarity, although all of these SXR regions have a similar light curve. In addition the peak spectral index for this event was 4.1 a little softer than the mean value.

Figure 8c shows the relation between the HXR and magnetogram. The brightest source is situated in the north and lies in the region of strongest magnetic field. However, there is too little data to establish if any time delay exists between the two HXR emission profiles.

Other than the strong source being in a region of strong magnetic field this flare behaves much the same as the others. However, the northern soft X-ray region is brighter than the southern and may be an indication that the acceleration site of the electrons responsible for this event may be closer to the north end. If that is the case, then it may be possible that the flaring electrons are able to penetrate deeper into the stronger region before being mirrored as there is less actual field convergence over a smaller section of loop. In addition the increased flight time to the other footpoint may mean a reduction in the number of electrons impacting the chromosphere in a focused region and causing high amounts of radiation.

In Table 3 we list the properties of all of the non-Sakao type events.

\section{Discussion}

This study has demonstrated that the relation between the magnetic flux density at the footpoints of a flaring loop and HXR emission is much more complicated than previously thought.

The most appropriate and fair method of measuring the magnetic flux density beneath any given HXR footpoint, in light of the data available, was determined. The method required each flare to be within $40^{\circ}$ of the solar disc centre and 
also data in the HXT $M 2$ channel was required to produce images free from a thermal component.

When one compares the actual intensity of any individual footpoint to the magnetic flux density beneath it across a large sample, it quickly becomes clear that there are other factors involved aside from the magnetic mirroring effect, not to mention the difficulties inherent in comparing flares of different magnitude. Although the magnetic field strength at the footpoints is likely a large factor in the majority of cases there must be other influences in some events. The magnetic connectivity is complex and although the SXT images show what appears to be a single loop, they only show structures containing hot plasma and give little information about any fine structure and the environment in which the flare occurs. This is especially important as it is the magnetic field convergence which is responsible for the mirroring of electrons and not the strength of the field alone. Therefore a more detailed study of the magnetic field surrounding the footpoints may give rise to a clearer understanding of these mirror points. If one studies the field around the footpoint, it may be possible to determine how much the field can expand and give an understanding of the level of convergence.

If one takes the HXR and mean magnetic field strength ratio between the two footpoints in each event, it begins to focus the problem by removing the issue of flare magnitude, and therefore effectively allowing one to compare a high magnitude event with a much lower magnitude. It was from this study that two main groups of flares were determined; the Sakao type (S-type) and the Non-Sakao type (N-type), where S-type events correspond to events where high HXR intensities which correspond to low mean magnetic field strengths and $\mathrm{N}$-type have low HXR with low magnetic field strength.

The uncertainties involved make the formation of a third class of event necessary, B-type. These were where the error bars cross the dividing line between the two classes above and as such are likely to have a more symmetrical nature or be dominated by measurement uncertainty.

From this sample of 32 events $43.8 \%$ were the traditional Sakao type, $25 \%$ were approximately symmetrical in mean magnetic field strength and $31.2 \%$ were the non-Sakao type.

There is little difference between the properties of each of these flares. The mean spectral index at the peak is almost the same in each of the three classes with a value of 3.4, and a standard deviation of 1.4 in the S-type, and 3.5, with a standard deviation of 1.2, in the N-type; with the soft-hard-soft evolution across the board. Therefore there is no quantitative difference which may have been an indicator of variations in the transport of electrons.

The 16th March 2000 event was an event which was part of the N-type sample. When one looks at the SXR image the northern region appears to consist of two parts. Both of these have very similar light curves consistent with the same plasma population and appear to be connected. It is not clear whether this is observed simply due to line of sight effects or whether they are separate loops. However, there is still a single HXR footpoint which is brighter than the southern source and is situated in a region of single polarity.

It is widely understood that the S-type events are the result of converging magnetic field lines in strong regions which act to mirror precipitating electrons and thus reduce the number available to form HXR through thick-target bremsstrahlung. Conversely with less convergence in weaker field regions, more electrons can precipitate to the chromosphere and gives rise to a stronger HXR footpoint. So why should we observe N-type events?

It was hoped that many of these events would have enough counts so that individual HXR light curves could be seen for each footpoint. This information could allow any time lags between emissions to be observed which would give weight to an asymmetry in the location of the acceleration site and thus the resulting precipitation of electrons and HXR emission at the footpoints. An asymmetry in the location of the acceleration site may be the answer to the occurrence of $\mathrm{N}$-type events. If the acceleration site is located closer to the brighter footpoint it may reduce the effects of convergence over the shorter distance, allowing more precipitation in this region than if the acceleration site was located at the loop apex. With further observations it may be possible confirm this, although we must not rule out the possibility of some other process impeding the electron precipitation at the weaker footpoint. The only two events in the N-type cases which did have timing information show simultaneous light curves for both footpoints, but there were few HXR images which were integrated over a relatively long period of time which gives rise to a poor cadence.

The B-type events determined earlier, enabled us to remove events which had the most influential uncertainties. These events were more or less symmetric in magnetic field strength at the footpoints, however the same could not be said for the HXR intensities. As a result the B-type events could not reliably be placed in S- or N-Type categories.

The work of Aschwanden et al. (1999) studied an asymmetric trap model. In this work it was assumed that the acceleration site for the electrons was equidistant from each of the flare footpoints and was thus at the loop apex. From this basis it was predicted that the median value for the ratio of the magnetic fields at the footpoints would be 1.2. We found in the case of the B-type events this is true, although the S-type have a median value of 0.4 and the $\mathrm{N}$-type a value of 3.4. Therefore, using our method of observation we were unable to confirm these predictions, although it is unknown how moving the acceleration site would affect their predictions.

\section{Conclusions}

The sample of 32 flare events has shown that it is not necessarily true that the stronger HXR footpoint is situated in the weaker magnetic field region. The weaker footpoint would appear to have a likelihood of 2:1 to be situated in a region of stronger magnetic field. Although the results of Sakao's thesis show a 4:1 relation, only 5 events were considered and the single observation which showed a N-type result was limited by the frequency of the ground based magnetograms. Our results show that these $\mathrm{N}$-type events occur more frequently than previously thought. There is little that distinguishes the groups of events and there is no quantitative difference between the peak spectral hardness across the groups, nor is there any real 
difference in the distribution and magnitudes of the HXR and magnetic ratios.

Acknowledgements. C.P.G. is grateful to PPARC for postgraduate student funding. L.v.D.G. was supported by the Hungarian Government grant OTKA T-038013. S.A.M. and L.v.D.G. would like to thank The Royal Society for funding through their Joint Project scheme. L.K.H. is grateful to PPARC for the award of an Advanced Felllowship. The authors thank Pascal Démoulin and Cristina Mandrini for inspiring discussion. The authors thank the MDI consortia, in addition to the Yohkoh/SXT and Yohkoh/HXT teams for their data. SOHO is a project of international cooperation between ESA and NASA. Yohkoh is a mission of the Japanese Institute for Space and Astronautical Science. We acknowledge the SURF for providing data for this publication. We would also like to thank the referee for helpful comments.

\section{References}

Asai, A., Masuda, S., Yokoyama, T., et al. 2002, ApJ, 578, L91

Aschwanden, M. J., Fletcher, L., Sakao, T., et al. 1999, ApJ, 517, 977

Berger, T. E., \& Lites, B. W. 2002, Sol. Phys., 213, 213

Gull, S. F., \& Daniell, G. J. 1978, Nature, 272, 686

Hagenaar, H. J., Schrijver, C. J., \& Title, A. M. 2002, ApJ, 584, 1107 Hanaoka, Y. 1996, Sol. Phys., 165, 275

Kosugi, T., Makishima, K., \& Murakami, T. 1991, Sol. Phys., 136, 17 Kosugi, T., Sakao, T., Masuda, S., et al. 1992, PASJ, 44, L45 Ogawara, Y., Takano, T., Kato, T., et al. 1991, Sol. Phys., 136, 10 Parks, G. K., \& Winckler, J. R. 1969, ApJ, 155 , L117

Sakao, T. 1994, Ph.D. Thesis, University of Tokyo Sato, J., Kosugi, T., \& Makishima, K. 1999, PASJ, 51, 127

Scherrer, P. H., Bogart, R. S., \& Bush, R. I. 1995, Sol. Phys., 162, 129 Tsuneta, S., Acton, L., \& Bruner, M. 1991, Sol. Phys., 136, 37 